\title{
Estrategias de intervención social mediante la vinculación comunitaria entre la Universidad Técnica Estatal de Quevedo y la Escuela de Educación Especial Pamuniq
}

\author{
Strategies for social intervention through community links \\ between the State Technical University of Quevedo and the \\ School of Special Education Pamuniq
}

\author{
Lcda. Inés Bajaña Mendieta ${ }^{1}$ \\ ibajana@uteq.edu.ec \\ Lcda. Marjori Torres Bolaños ${ }^{1}$ \\ ttorres@uteq.edu.ec \\ Ing. Marisol Veliz Aguilar ${ }^{1}$ \\ mveliz@uteq.edu.ec Lcda. \\ Verónica Osorio ${ }^{1}$ \\ aosorio@uteq.edu.ec \\ Ing. Javier Gómez Pianda ${ }^{1}$ \\ javagop@gmail.com Lcda \\ Rosmaira Martinez ${ }^{1}$ \\ rmartinez@uteq.edu.ec
}

Recibido: 1/07/2018, Aceptado: 1/09/2018

\begin{abstract}
RESUMEN
Siendo parte de una institución de educación superior ubicada en un cantón con alto índice de discapacidad y comprobando que consiste un escaso conocimiento e involucramiento de los estudiantes universitarios, la Universidad Técnica Estatal de Quevedo firmó en el año 2010 un convenio marco con el objetivo de mejorar los espacios de educación y desarrollo de potencialidades de los niños y adolescentes de la escuela especial del Patronato Municipal de la Niñez Quevedeña (Pamuniq) propiciando acciones de participación comunitaria con los estudiantes de la Carrera de Ingeniería en Diseño Gráfico y Multimedia. Para alcanzar los objetivos se realizaron las siguientes fases: Diagnóstico del Problema, identificación aliados estratégicos, elaboración de propuesta, reconocimiento del sitio y espacios de la institución beneficiaria, selección de estudiantes participantes, socialización del Proyecto de Vinculación, ejecución y evaluación de resultados. El estudio corresponde a la metodología cualitativa aplicando además los métodos de la investigación-acción y método de proyectos. Los proyectos de vinculación e integradores ejecutados, propiciaron acciones solidarias fomentando la práctica de valores humanos y la inclusión social.
\end{abstract}

\footnotetext{
1 Docentes de la Universidad Técnica Estatal de Quevedo. Ecuador
} 


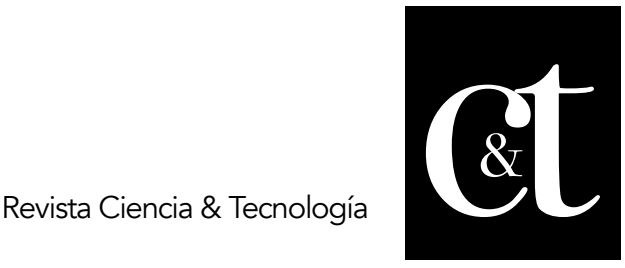

No. 20, 31 de octubre de 2018

ISSN impreso: 1390 - 6321

Palabras clave: inclusión, educación especial, estrategias, vinculación, universidad

\section{ABSTRACT}

The State Technical University of Quevedo through the signing of a Framework Agreement in 2010 establishes actions in the construction of an inclusive society from the perspective of local disability by applying social inclusion strategies with the aim of improving educational spaces and development of potentialities of children and adolescents of the special school of the Municipal Patronage Board of Children Quevedeña (Pamuniq) promoting solidarity actions of community participation with university students of the Engineering Career in Graphic Design and Multimedia through linking projects. The following activities were developed to reach the objectives: problem diagnosis, identification of strategic allies, elaboration of proposal, recognition of premises of the beneficiary entity, selection of participant students, socialization of the linking project, execution and evaluation of results. The study used qualitative methodology. The linking and integrating projects allowed solidarity actions to promote the human values and social inclusion.

Keywords: inclusion, special education, strategies, linking, university

\section{Introducción}

La Universidad Técnica Estatal de Quevedo en concordancia con la misión institucional de "Formar profesionales y académicos competitivos y de excelencia; generando conocimiento, tecnología, servicios de calidad, soluciones a los problemas de la sociedad; sustentada en principios y valores éticos". Promoviendo a través de la línea de investigación educativa un espacio estructurado de conocimiento y metodología, donde confluyen diferentes acciones de los grupos de investigación que trabajan en colaboración con las instituciones vinculadas a la UTEQ.

Nuestra universidad asume con responsabilidad social desde el año 2010 el reto de contribuir a través de proyectos de vinculación con el mejoramiento de los procesos educativos de La Escuela Especial del Patronato Municipal de la Niñez Quevedeña (Pamuniq) Institución constituida el 1 de julio de 1988 con el propósito de atender las necesidades de los niños con discapacidad, una población invisibilizada dentro del sistema educativo nacional. En la actualidad el enfoque de inclusión ha ido ganando terreno en los ámbitos educativo y social, cuyo principal propósito es hacer frente a los altos índices de exclusión, discriminación y desigualdad educativa.

En la Provincia de Los Ríos y de manera particular en el Cantón Quevedo, el nacimiento de niños con discapacidad presenta altos porcentajes. Según el Registro del Consejo Nacional para las Discapacidades (CONADIS), remitiéndose a la fuente del Ministerio de Salud Pública hasta mayo del 2018 existen en Quevedo 4.305 personas con discapacidad. Identificando los siguientes tipos de discapacidad: Física $56,24 \%$, intelectual $21,63 \%$, auditiva $10,24 \%$.visual $8,8 \%$. psicosocial $3,09 \%$.

102 


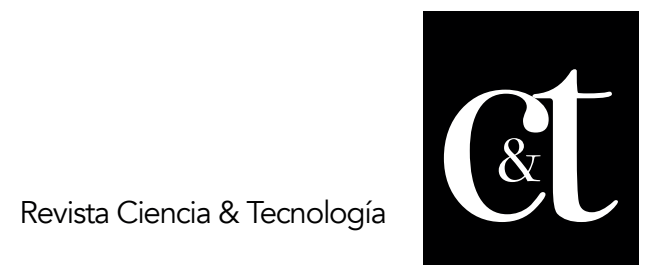

No. 20, 31 de octubre de 2018

ISSN impreso: 1390 - 6321

El Patronato Municipal del Niño de Quevedo es la única institución que brinda servicios especializados a esta población y a otros cantones que confluyen en Quevedo por carecer de entidades que brinden una atención especializada. Las investigaciones realizadas por la Misión Manuela Espejo, Programa lanzado en el año 2009 y ejecutado por la Secretaria Técnica de Discapacidades (SETEDIS), detectó en el primer barrido a 12.868 personas con discapacidad en Ecuador de los cuales 404 eran casos críticos situados en la Provincia de Los Ríos correspondiendo 70 de ellos a Quevedo. Entre las causas que originan la discapacidad a nivel de la Provincia constan: Enfermedad adquirida 8.454, Congénito genético 5.733 accidente de tránsito 972, problemas de parto 433 (CONADIS, 2013)

La educación es un derecho que los Estados han de promover, respetar y garantizar a todas las personas a lo largo de su vida. Hacer efectivo este derecho supone que todos tengan acceso a una educación de calidad en igualdad de oportunidades y condiciones (Maturana, 2010). Justamente estos elementos son los que definen a la educación inclusiva, que busca reducir toda forma de discriminación y exclusión (Arnaiz, 2012). Por ello consideramos importante emprender acciones conjuntas con la Universidad para difundir información en temas relacionados, con Prevención de discapacidad difusión de los Derechos de las personas especiales, capacitación en los talleres ocupacionales para que puedan ejercer sus derechos a plenitud.

Cronología de la Inclusión Educativa

La Clasificación Internacional del Funcionamiento, de la Discapacidad y de la Salud (CIF) define la discapacidad como un término genérico que abarca deficiencias, limitaciones de la actividad y restricciones a la participación. Se entiende por discapacidad la interacción entre las personas que padecen alguna enfermedad, entre ellas: Parálisis cerebral, síndrome de Down y depresión, factores personales y ambientales (Di Nasso, 2010). Se calcula que más de mil millones de personas es decir, un $15 \%$ de la población mundial están aquejadas por la discapacidad en alguna forma. Tienen dificultades importantes para funcionar entre 110 millones $(2,2 \%)$ y 190 millones $(3,8 \%)$ personas mayores de 15 años. Además, las tasas de discapacidad están aumentando debido en parte al envejecimiento de la población y al aumento de la prevalencia de enfermedades crónicas (Valencia, 2014).

La lucha por una educación inclusiva y de calidad se basa en el derecho de todos y todas a recibir una educación que promueva el aprendizaje durante toda la vida. Un sistema educativo es de calidad cuando presta atención a los grupos marginados y vulnerables y procura desarrollar su potencial (UNICEF, 2013).

A nivel mundial se han establecido acuerdos y compromisos para que los países asuman responsabilidades a favor de una educación inclusiva:

En 1936 La Constitución de la URSS contaba con un artículo el $120^{\circ}$ que establecía 
que "los ciudadanos tienen derecho a la asistencia económica en la vejez, así como en caso de enfermedad y de pérdida de la capacidad de trabajo". En el caso particular de las personas con discapacidad se garantizaba una pensión de por vida y la gratuidad de todos los tratamientos (Goldman, 2010).

En 1948, la Declaración Universal de los Derechos Humanos en su artículo 26 reconoce que todas las personas tienen derecho a la educación. Desde ese momento se recorre un largo camino y se han hecho grandes esfuerzos para alcanzar ese objetivo.

En 1978 el Informe Warnock, elaborado por Mary Warnock para Inglaterra, Escocia y Gales, publicado en 1978 determina que la educación es un bien al que todos tienen derecho. El informe establece que la educación especial debe tener un carácter adicional y no paralelo, por lo que las instituciones de educación especial deben seguir existiendo para educar a estudiantes con graves y complejas discapacidades.

En 1982 la Asamblea General de las Naciones Unidad aprobó el Programa de Acción Mundial para los Impedidos, con el fin de promover medidas eficaces para la prevención de la discapacidad. En este programa se defiende por primera vez a la discapacidad como la relación que existe entre las personas y su entorno, y se adoptan los principios de normalización, integración y participación. En el año de 1990 La Declaración Mundial sobre Educación para Todos efectuada en Jomtien, Tailandia (1990) conocida como "Satisfacción de las Necesidades Básicas del Aprendizaje", señala a la educación como un derecho fundamental de todos los hombres y mujeres de todas las edades del mundo.

La Asamblea General de las Naciones Unidas en 1992, declara el 3 de diciembre de cada año, Día Internacional de los Impedidos y hace hincapié una vez más en la importancia de la integración plena de los discapacitados en la sociedad.

En 1993 se adoptan las Normas Uniformes sobre la igualdad de oportunidades para las personas con discapacidad, las cuales se centran en lograr la igualdad de participación en diferentes esferas y fomentando la aplicación de medidas y mecanismos de supervisión.

En 1994 los temas y acuerdos tratados en el Informe Warnock fueron aceptados a nivel internacional en varios documentos, especialmente en la Declaración de Salamanca, en la Conferencia Mundial sobre Necesidades Educativas Especiales: "Acceso y Calidad", en la que se establecen los principios, políticas y prácticas para favorecer la integración de los estudiantes con necesidades educativas especiales en las escuelas regulares.

En el Foro Mundial de Educación para Todos en Dakar en el año 2000, se reafirma la idea de la Declaración Mundial sobre Educación para Todos, respaldada por la 


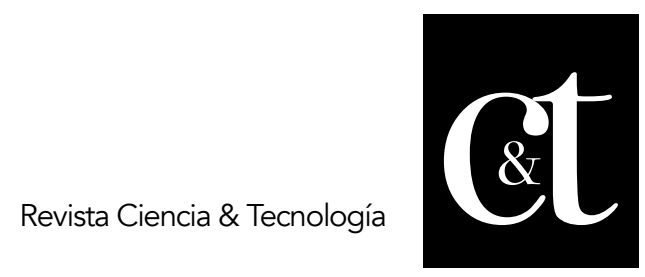

No. 20, 31 de octubre de 2018

ISSN impreso: 1390 - 6321

Declaración Universal de Derechos Humanos y la Convención sobre los Derechos del Niño, en su condición de seres humanos, tienen derecho a beneficiarse de una educación que satisfaga sus necesidades básicas de aprendizaje.

La Asamblea General de las Naciones Unidas aprobó la convención el 13 de diciembre de 2006, y se abrió a la firma el 30 de marzo de 2007. Los Estados que la ratifican están jurídicamente vinculados a respetar las disposiciones que en ella se recogen, mientras que para los demás Estados, la convención constituye una norma internacional que deben esforzarse por respetar. El Ecuador fue el país número veinte en adherirse a la convención el 30 de marzo del 2007, que fue ratificada por la Asamblea Nacional Constituyente el 3 de abril de 2008.

Por primera vez en la historia republicana del Ecuador la Constitución garantiza de forma transparente el derecho de las personas con discapacidad a ejercer una vida plena. Art. 48.- El Estado adoptará a favor de las personas con discapacidad medidas que aseguren:

1. La inclusión social, mediante planes y programas estatales y privados coordinados, que fomenten su participación política, social, cultural, educativa y económica.

2. El establecimiento de programas especializados para la atención integral de las personas con discapacidad severa y profunda, con el fin de alcanzar el máximo desarrollo de su personalidad, el fomento de su autonomía y la disminución de la dependencia.

El Ministerio de Educación en el año 2010, desarrolló un proceso de reestructuración, desde una nueva propuesta organizativa y curricular en todos los niveles y modalidades. Ésta permitirá fortalecer la educación inclusiva para todos los grupos de atención prioritaria.

En el 2011 se aprueba la Ley Orgánica de Educación Intercultural, en la cual se hace efectivo el derecho a la educación que tienen las personas con discapacidad, garantizando la inclusión de éstas a los establecimientos educativos dentro del marco del Buen Vivir, la interculturalidad y la plurinacionalidad, basado en la relación de todos los actores sociales y la comunidad educativa. En 2012 inició un proceso de estandarización y todas las Unidades Educativas del Milenio poseen infraestructura similar.

El Consejo Nacional de Discapacidades (CONADIS), por competencia legal, descrita en la Constitución de la República, Sexta Transitoria y Artículo 156, en el año 2013 coordinó la construcción de la Agenda Nacional para la Igualdad en Discapacidades (ANID) 2013-2017. 


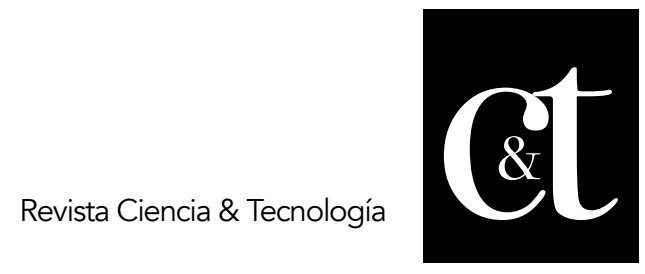

No. 20, 31 de octubre de 2018

ISSN impreso: 1390 - 6321

En el Año 2017 con el fin de aumentar sus estándares en cuanto calidad y acceso a derechos, el gobierno fortalecerá la educación inclusiva para lo cual se desarrolló un protocolo para la inclusión de personas con discapacidad que permitirá la aplicación de esta política pública hasta el año 2020.

En 2018, el MINEDUC - Ministerio de Educación Subsecretaría de Educación Especializada e Inclusiva y la Dirección Nacional de Educación Especial e Inclusiva Proyecto: K007 MINEDU han presentado el Proyecto Modelo de Educación Inclusiva con el objetivo de fortalecer y mejorar la atención en educación especializada y la inclusión educativa en educación ordinaria, para niños y jóvenes con necesidades educativas especiales asociada o no a la discapacidad.

Derecho a la Inclusión

En Ecuador, haciendo una revisión retrospectiva sobre del tema encontramos que de acuerdo al VI Censo de Población y V de Vivienda (INEC, 2001), el porcentaje de la población ecuatoriana que señala tener discapacidad fue de $4.7 \%$, datos que en el transcurso de una década ascendió a 5.6\%, según los datos del VII Censo de Población y VI Vivienda (INEC, 2010). Según el CENSO 2010 en el país existen 136.481 personas en edad escolar que tienen algún tipo de discapacidad; de esta población, el Sistema educativo Nacional está atendiendo a 10.103 estudiantes (7\%) en educación especializada y 16.146 estudiantes (12\%) en educación inclusiva. Adicional a ello, existen alrededor de 34.117 (25\%) estudiantes que están siendo atendidos tanto en Fundaciones, como ONG's y otros; sin embargo, todavía existe un $56 \%$ del total de la población que no está siendo atendida. Actualmente, se encuentran capacitados 17.645 docentes en educación especial e inclusiva, proyectándose para el presente año lectivo un incremento del $11 \%$ partiendo del índice de crecimiento proyectado para todos los servicios educativos especializados e inclusivos.

Subestimar el potencial de las personas con discapacidad es uno de los factores que más atentan contra su inclusión y su disfrute de la igualdad de oportunidades. Las actitudes negativas se dan en toda la sociedad, desde los profesionales, políticos y otros responsables de tomar decisiones hasta las familias y los compañeros, así como las propias personas con discapacidad que, ante la ausencia de pruebas de que se les valora y apoya en su desarrollo, subestiman a menudo sus propias capacidades (CONADIS, 2013).

La educación inclusiva se apoya en la convicción de que todos los niño/as pueden aprender cuando se les otorgan las oportunidades de aprendizaje apropiadas y si se planifica el aprendizaje individualizado; se crean equipos de apoyo; se estimulan las capacidades y responsabilidades sociales entre los niño/as; se evalúa el rendimiento en programas de infantes ( 0 a 5 años); se planifica la transición de una etapa de la educación a la siguiente; se trabaja en colaboración con los padres y otros miembros de la comunidad; se aplican planes de formación del personal y existe responsabilidad 


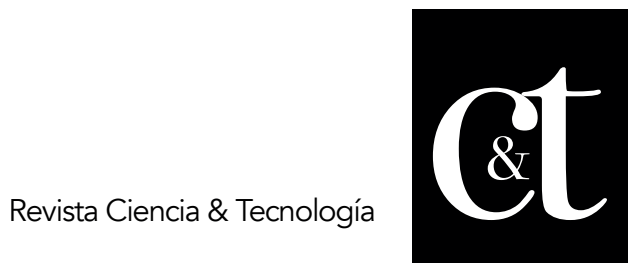

No. 20, 31 de octubre de 2018

ISSN impreso: 1390 - 6321

por la gestión (Barrera y Quiñones, 2009; Solórzano, 2013).

\section{Educación Especial}

La Educación Especial es una modalidad de atención del sistema educativo de tipo transversal e interdisciplinario dirigida a estudiantes con necesidades educativas especiales asociadas a la discapacidad no susceptible de inclusión. Educación Especial es entendida como un proceso educativo dinámico que reconoce y atiende la diversidad del alumno y en la que se apoya para permitir a éste la consecución de metas más ajustadas a sus características personales (Riaño, De la Rosa y Bermúdez, 2010). Plantea el reconocimiento de las diferencias y el respeto de la diversidad, asegura el cumplimiento del principio de equiparación de oportunidades, como un aspecto de enriquecimiento. Se caracteriza por proveer un conjunto de servicios, recursos humanos y técnicos, conocimientos especializados y ayudas, con el propósito de asegurar aprendizajes de calidad a los estudiantes (Ministerio de Educación, s.f.).

La Educación Especial sirve como base educativa y preparativa para una posible inclusión de los estudiantes, así lo determina el Ministerio de Educación del Ecuador, que señala lo siguiente: Accederán a estas instituciones los estudiantes con necesidades educativas especiales asociadas a discapacidad, que determine la evaluación del equipo de la Unidad Distrital de Apoyo a la Inclusión (UDAI).

\section{Vinculación con la Comunidad}

La Constitución de la República del Ecuador señala que "La educación se centrará en el ser humano y garantizará su desarrollo holístico, en el marco del respeto a los derechos humanos, al medio ambiente sustentable y a la democracia; será participativa, obligatoria, intercultural, democrática, incluyente y diversa, de calidad y calidez; impulsará la equidad de género, la justicia, la solidaridad y la paz; estimulará el sentido crítico, el arte y la cultura física, la iniciativa individual y comunitaria, y el desarrollo de competencias y capacidades para crear y trabajar".

Sensibilizar a la sociedad quevedeña sobre los derechos de las personas con discapacidad y el respeto a su dignidad, a fin de erradicar progresivamente toda forma de discriminación así como de eliminar las barreras culturales y actitudinales que limiten su desarrollo y plena inclusión. La Constitución de la República (2008), el Plan Nacional para el Buen Vivir 2013-2017, la Convención interamericana para la eliminación de todas las formas de discriminación contra las personas con discapacidad y la Convención sobre los derechos de las personas con discapacidad.

Una sociedad incluyente debe promover la integración social en el marco del respeto de los derechos de las personas, especialmente el derecho a la vida, al desarrollo y a la participación. A la educación superior le corresponde crear espacios de convergencia entre los diferentes actores educativos, el sistema político-estatal, nacional, regional y local, los sectores productivos, los sectores sociales y culturales para articular necesidades, perspectivas, visiones y horizontes de futuros posibles. 


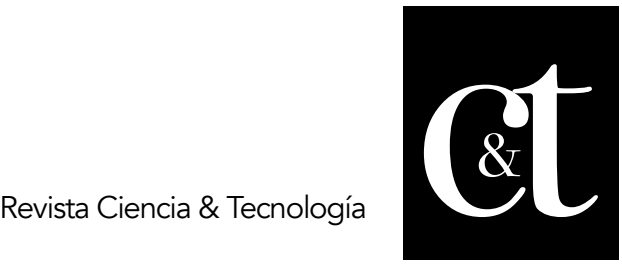

No. 20, 31 de octubre de 2018

ISSN impreso: 1390 - 6321

(Heredero, 2008).

El Rol de la Universidad

La estrategia principal radica en que la educación superior dinamice sistemas y actorías para la producción y ejecución de proyectos que transfieran el conocimiento de forma colaborativa, creativa y solidaria, e intrínsecamente permitan definir su identidad con lo público. Estas actorías sociales, productivas, culturales y políticas, nos convocan a tomar en cuenta el fortalecimiento de la institucionalidad, la cohesión social, la ciudadanía deliberativa, y restitución de derechos (Papanek, 1977).

Edgar Morín, al respecto nos invita a "reconocer que todo sujeto es potencialmente, no solo actor sino autor, capaz de cognición, elección y decisión" (2003:89), y por ello es importante que el currículo a través de las prácticas de servicio a la comunidad promueva el ejercicio de aprendizajes éticos.

La dinámica entre la racionalidad afectiva de Morín (2003) que tiene como escenario fundamental los aprendizajes curriculares y experienciales, el mundo de la vida de Habermas que plantea la interacción cultura, sociedad e identidad y el de Husserl (1988) que aporta con una praxis que permite la construcción del sentido en el proceso de implicación del sujeto educativo con los contextos que es capaz de crear y recrear en función de su experiencia de producción del conocimiento y de constitución de su proyecto de vida resultando enriquecedora la interrelación con las personas con discapacidad desarrollando Proyectos de vinculación social para promover la inclusión de niños, niñas y adolescente con discapacidad de la Escuela de Educación Especial Pamuniq del Cantón Quevedo.

Inteligencias múltiples del diseñador gráfico

La Formación basada en Competencias es el modelo curricular adoptado por la UTEQ plantea que las competencias son procesos complejos de desempeño ante problemas con idoneidad y compromiso ético, y se enmarcan en la formación integral que demanda el direccionamiento estratégico de una malla curricular multidisciplinaria integradas por módulos, proyectos integradores, las prácticas comunitarias y prácticas pre profesionales condición didáctica que permite cumplir el principio de la sistematicidad de la enseñanza. Desde este enfoque curricular la teoría y práctica van edificando el perfil del futuro diseñador que tiene en ventaja competitiva generar diversidad de composiciones en formas, tonos o matices, tamaños y distribución espacial.

Los campos de la ciencia han acogido a la creatividad como un factor influyente a su proceso de enseñanza-aprendizaje promoviendo la conformación de equipos multidisciplinario en pertinencia a la formación del diseñador gráfico y multimedia y a los requerimientos de una sociedad demandante. El concepto de Inteligencias Múltiples desarrollado por el psicólogo estadounidense Howard Gardner durante la década de los años 80 establece que las capacidades de nuestra mente no forman 


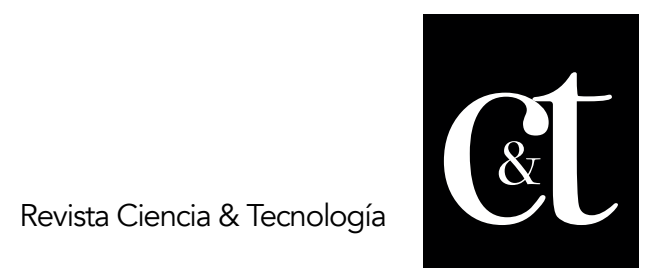

No. 20, 31 de octubre de 2018

ISSN impreso: 1390 - 6321

parte de una sola habilidad Ilamada inteligencia, sino de ocho que trabajan en paralelo: Lingüística, lógico-matemática, espacial, musical, corporal, intrapersonal, interpersonal y naturalista. Acogiendo esta teoría se establecen proyectos que vinculen al estudiante con una realidad social donde interiorizar el modo de explorar y procesar la información para desarrollar un proceso creativo en el converge el razonamiento numérico, lógico y estructural, el uso efectivo de las palabras en expresión a través de la voz y del texto, el lenguaje corporal para transmitir ideas, sentimientos mediante observación, y reflexión para transmitir pensamientos y actitudes ante un entorno socio-cultural globalizado.

Situación de la Institución beneficiaria Pamuniq

La Escuela de Educación Especial Pamuniq cuenta con una acogedora infraestructura, los niños y jóvenes que asisten a la Escuela reciben atención médica y alimentación. Los Tipos de discapacidades que poseen los escolares son: Síndrome de Down, parálisis cerebral, discapacidad intelectual (leve, moderada), de igual manera de aprendizaje, hipoacusias, autismo, hidrocefalia, microcefalia. Tiene tres niveles de Educación inicial, básica y desde Abril del 2010 los Talleres ocupacionales para los adolescentes. Con el propósito de promover una rehabilitación integral en los niños con discapacidad, brinda atención especializada en: Psicología, medicina general, trabajo social, rehabilitación física, fisiatría, terapia de lenguaje, estimulación temprana, odontología, terapia ocupacional, laboratorio clínico y farmacia. Mediante la observación participante y diálogos con docentes de la Escuela Pamuniq, además del análisis de la información publicada en los medios de comunicación se identificaron los siguientes problemas en la educación especial local:

- Escaso conocimiento e involucramiento de los estudiantes universitarios con la comunidad para incentivar el buen trato y ejercicio de los derechos de las personas con discapacidad.

- Insuficiente coordinación y articulación de organizaciones e instituciones públicas y privadas en temas de sensibilización y respeto de los derechos a los grupos de atención prioritaria.

- Escasos recursos didácticos especializados para la enseñanza en educación especial. Los programas educativos no han sido adaptados para los diferentes tipos de discapacidad.

- Insuficiente presupuesto económico de Pamuniq para posicionamiento de imagen corporativa que difunda los servicios que oferta a la comunidad.

- Gran porcentaje de adolescentes con discapacidad entre 14 y 18 años sin oportunidades de inserción laboral.

\section{Metodología}

Los enfoques son diversos, pero la meta de este proyecto ha sido, la comprensión en profundidad del proceso de inclusión y capacitación ocupacional. Por ello, se ha optado por la utilización de una metodología cualitativa entendida como "Una actividad sistemática orientada a la comprensión en profundidad de fenómenos 


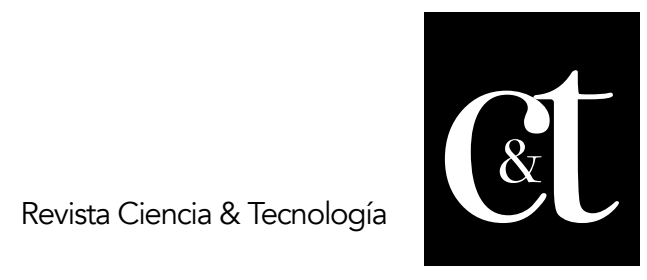

No. 20, 31 de octubre de 2018

ISSN impreso: 1390 - 6321

educativos y sociales".

Aplicamos el método de la investigación-acción puesto que se inicia como una idea colectiva que contribuirá a la mejora en el proceso enseñanza aprendizaje de Pamuniq, integrando en la fase inicial indagación y diálogo con los alumnos participante y profesores de la escuela de educación especial desarrollando de manera secuencial los siguientes pasos: Delimitación del problema a investigar en la acción, la planificación del proceso de acción, la ejecución de la acción y la evaluación de lo generado en la acción. Analizar con base en la evaluación del plan los resultados alcanzados al culminar las prácticas de vinculación quedan registrados en el informe final que presentan los universitarios donde narran las acciones de las actividades cumplidas en las 160 o 240 horas establecidas en el Reglamento interno, incluyendo además la ficha de observación, reportes mensuales, encuestas, entrevistas, registro fotográficos de lo que ocurre en los procesos educativos implicados en la acción y grabaciones en cintas videos de los eventos en la acción.

El docente coordinador de servicios la comunidad, presenta al finalizar el semestre un informe analítico en el que se recoge de manera sistemática su opinión sobre la información obtenida de los datos y presentación de resultados de los grupos de trabajo integrado para tal propósito. Utilizando un enfoque interpretativo desde una perspectiva social y humanista, atribuyendo un significado a lo observado para descubrir el sentido que tiene para el universitario la experiencia de servir a la comunidad más necesitada.

Método de proyectos

El método de proyectos, garantiza el desarrollo de las capacidades para operar con las diferentes disciplinas, y a trazar estrategias correctivas para dar solución al problema planteado. Se parte del interés del alumnado para abordar cuestiones que estos se esbozan y que nacen esencialmente de la vida habitual. Está cimentado en la idea de que el conocimiento se erige de manera social y, por tanto, se circunscribe epistemológicamente en las teorías socio constructivista del aprendizaje.

Etapas aplicadas en el proyecto:

- Diagnóstico del Problema

- Determinar aliados estratégicos.

- Elaboración de Propuesta.

- Reconocimiento del sitio y espacios de la institución beneficiaria.

- Socialización del Proyecto de Vinculación fase No 1.

- Ejecución.

- Evaluación de Resultados.

Contenidos Trabajados:

- Teóricos. Preparar contenidos gráficos con imágenes del currículo para que los 


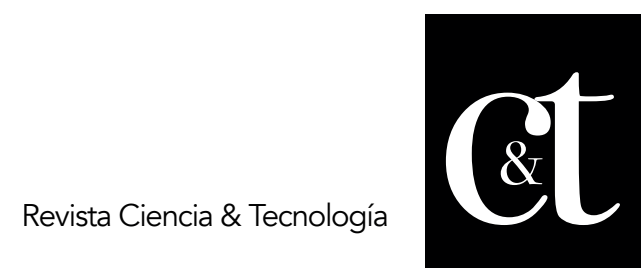

No. 20, 31 de octubre de 2018

ISSN impreso: 1390 - 6321

alumnos comprendan, un gran porcentaje de jóvenes de los talleres no leen ni escriben.

- Procedimentales. Enseñar mediante la praxis todos los procesos requeridos

- Actitudinales. En este contexto educativo interactúan los estudiantes de la Universidad quienes se sensibilizan y demuestran sus conocimientos de manera práctica y solidaria promueven la inclusión y respeto capacitando al alumno del PAMUNIQ.

\section{Resultados}

Los académicos y estudiantes de la Universidad Técnica Estatal de Quevedo, sensibles a la problemática de las personas con adversidad funcional, durante el periodo 20102017 construyen junto a docentes y directivos del Pamuniq estrategias de inclusión social mediante los proyectos de vinculación y los proyectos integradores de cada módulo de la Carrera de Ingeniería en Diseño Gráfico y Multimedia, con la finalidad de realizar capacitaciones en ciclos permanentes en áreas como: Computación, dibujo artístico, pintura, arte y cultura, durante estos años, participaron de exposiciones, festivales, encuentros deportivos y varias celebraciones especiales planificadas, que informaron a la ciudadanía quevedeña del trabajo emprendido de manera conjunta por ambas instituciones. Los alumnos de los talleres ocupacionales elevaron su autoestima gracias a la presencia de los estudiantes universitarios que participaron como capacitadores, generando una empatía que permitió a los dos grupos integrarse con mayor confianza a los procesos de capacitación, mejorando así su perspectiva de vida.

Los docentes de la Escuela aplican en su ejercicio profesional el currículo de Educación Especial, apoyándose con los recursos didácticos elaborados por los estudiantes en apoyo al proceso de mejoramiento de enseñanza-aprendizaje. En el contexto de esta experiencia, se han promovido valores como: inclusión, respeto, solidaridad, tolerancia, amistad, cortesía, justicia, equidad, perseverancia, cooperación y superación. 


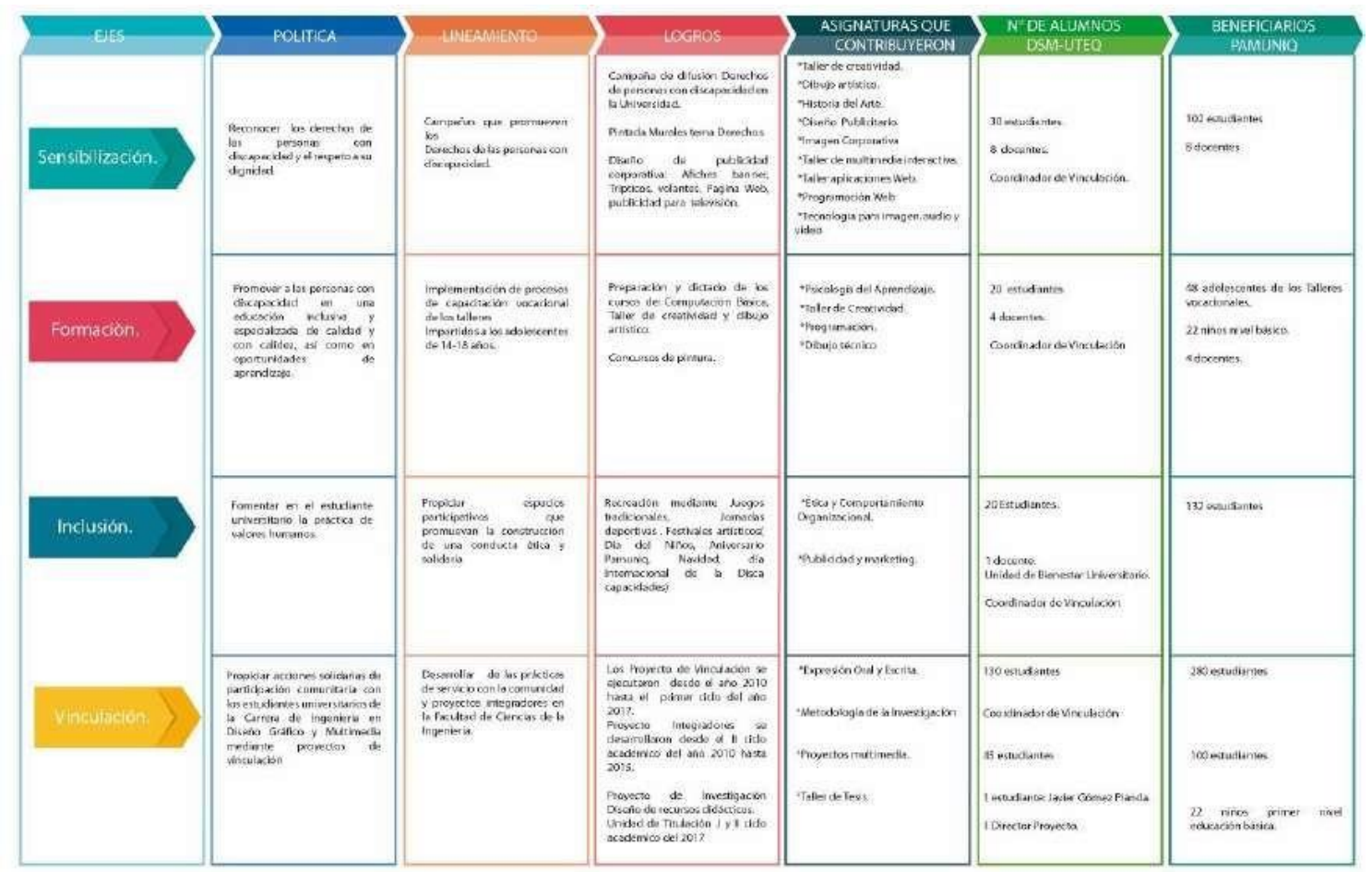

Gráfico 1. Proceso de mejoramiento de enseñanza-aprendizaje

Fuente: Elaboración propia.

\section{Conclusiones}

En la enseñanza Superior se debe trabajar con un sistema en valores que garantice educar al estudiante en criterios propios, en una cultura del comportamiento social, capaces de actuar con estilo de vida ético y democrático, que hagan posible su realización personal y social .Consideramos que esta experiencia transforma positivamente la vida de un estudiante universitario, que aprende a valorar a las personas más allá de sus diferencias, para los alumnos de Pamuniq compartir con jóvenes de su misma edad ha permitido que aprendan a socializar sin miedo, ni temores a ser rechazados elevando su autoestima.

En cada actividad emprendida se evidenció el entusiasmo, alegría de ambos grupos de actores. La Universidad Técnica Estatal de Quevedo promueve la vinculación responsable del estudiante universitario para que en el proceso formativo adquiera competencias que le permitan planificar, organizar, dirigir, coordinar y desarrollar programas con los sectores sociales, más vulnerables acorde a lo establecido en el "Plan Nacional de Buen Vivir ". A través de fomentar un aprendizaje integral en el estudiante de esta Carrera se promueve lo que es una tendencia del mercado conocido como Diseño Socialmente Responsable categoría que centra su atención en 


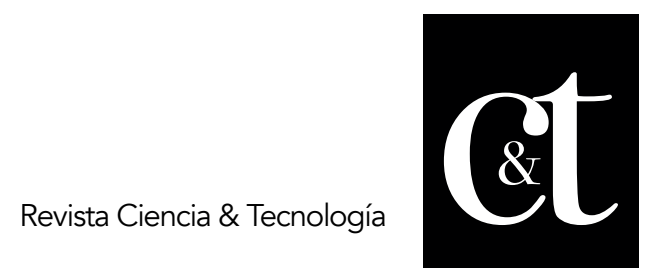

No. 20, 31 de octubre de 2018

ISSN impreso: 1390 - 6321

cómo se diseña, con qué se diseña, los efectos sociales, económicos, políticos, culturales y ambientales que generan las propuestas de diseño en su producción, uso y desuso. Este concepto se caracteriza por tener una actitud de respeto y de servicio por el bien del desarrollo de las personas, por la promoción de proyectos que incentiven la convivencia justa entre la gente, por propiciar acciones que disminuyan la violencia, por buscar una sociedad y por la formación de ciudadanos cívicos.

Es fundamental que toda la sociedad ecuatoriana y sus instituciones públicas y privadas redefinan novedosas estrategias de intervención inclusivas formativas de manera que las personas con capacidades especiales puedan acceder y conservar un empleo digno esto contribuye a desarrollar su autonomía e independencia.

\section{Referencias bibliográficas}

Arnaiz, P. (2012). Escuelas eficaces e inclusivas: cómo favorecer su desarrollo. Educación Siglo XXI, [S.I.]. V. 30, No.1. pp. 25-44. Recuperado de: http://revistas.um.es/educatio/article/view/149121/132111. Fecha de acceso: 24 jun. 2018

Barrera Jurado, G. S. y Quiñones Aguilar, A. C. (2009). Diseño socialmente responsable: ideología y participación. Bogotá: Pontificia Universidad Javeriana, Facultad de Arquitectura y Diseño.

CONADIS (2013). Agenda Nacional para la Igualdad 2013-2017.

Di Nasso, P (2010). Mirada histórica de la discapacidad. Fundación Cátedra Iberoamericana, Universitat de les Illes Balears, Recuperado de: www.uib.es/catedra_iberoamericana.

Goldman, W (2010). La Mujer, el Estado y la Revolución, Buenos Aires, Instituto de Pensamiento Socialista (IPS).

Heredero, E. (2008). A la calidad de la educación desde los espacios escolares: Visión del profesorado y perspectivas en la educación castellano - manchega. España: Universidad de Alcalá.

Husserl, E. (1988). Las conferencias de Paris, México, UNAM. (Pronunciadas en 1929 y publicadas por primera vez en alemán en 1950)

INEC (2001). VI Censo de Población y V Vivienda.

INEC (2010). VII Censo de Población y VI Vivienda.

Maturana, H. (2010). El Sentido de lo Humano. Buenos Aires: GRANICA.

Ministerio de Educación. (s.f.). Instituciones de Educación Especial. Recuperado de: https://educacion.gob.ec/instituciones-de-educacion-especial.

Morín, E. (2003). Introducción al pensamiento complejo, Barcelona, Gedisa.

Papanek, V. J. (1977). Diseñar para el mundo real: ecología humana y cambio social. 


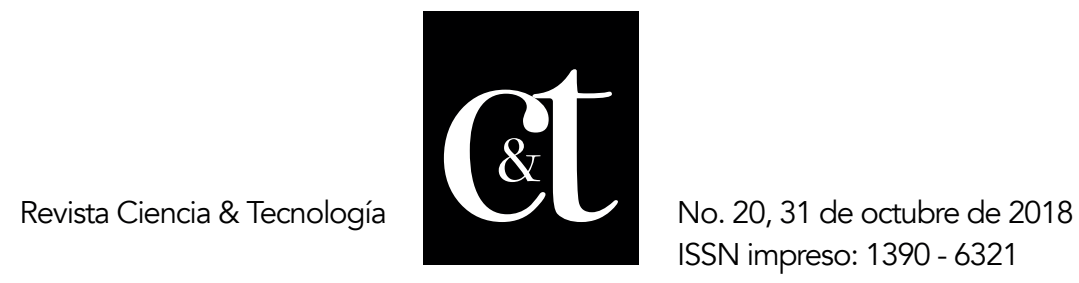

Madrid, España: Ed. Blume.

Riaño, C., De la Rosa, J. y Bermúdez, D. (2010). Metodologías para eldiseño de cartel social desde América Latina. Bogotá: Universidad Nacional de Colombia.

Solórzano, M. (2013). Espacios accesibles en la escuela inclusiva. Revista Electrónica Educare, V. 17, No. 1, pp. 89-103.

UNICEF (mayo 2013) Estado Mundial de la Infancia Niños y Niñas con Discapacidad. UNICEF. Niñas y Niños con Discapacidad. Resumen Ejecutivo. New York, New York, USA: UNICEF.

Valencia, L. (2014). Breve Historia de las personas con discapacidad. De la Opresión a la Lucha por sus derechos. Recuperado de: https://rebelion.org/docs/192745.pdf 


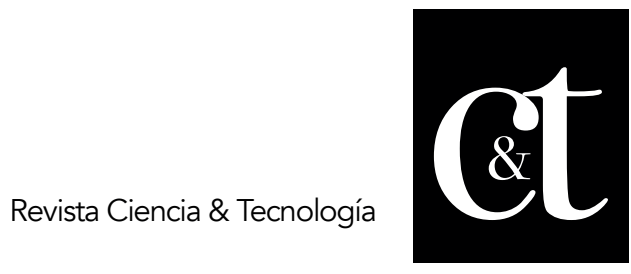

No. 20, 31 de octubre de 2018

ISSN impreso: 1390 - 6321

\section{ANEXOS \\ Estudiantes de la Carrera Ingeniería en Diseño Gráfico y Multimedia comparten conocimientos. (2010)}

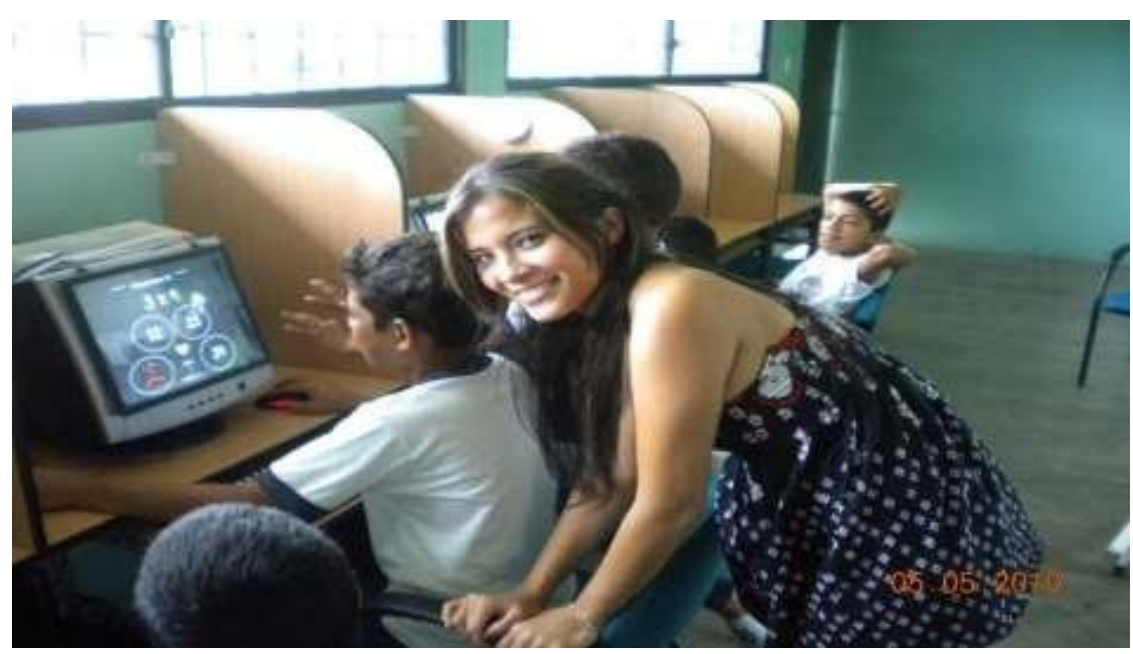

Los adolescentes reciben Talleres computación

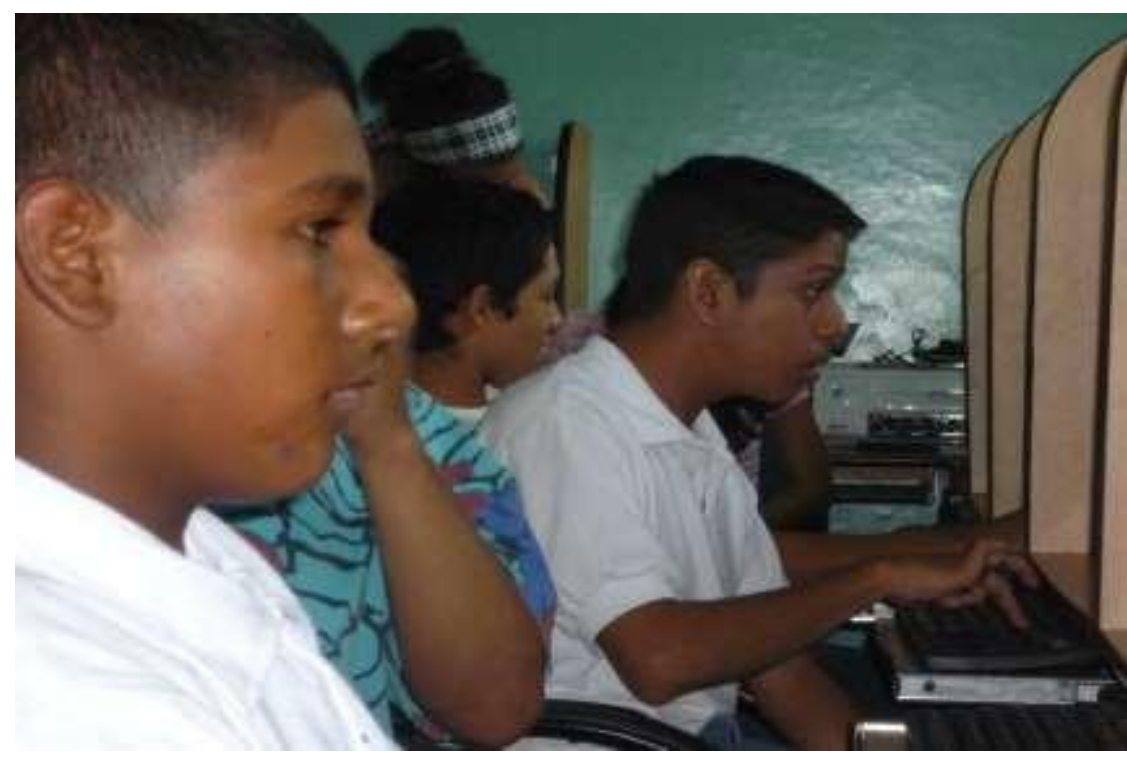

Jóvenes de los talleres ocupacionales en clase de computación básica. 


\section{UTEQ APOYANDO A PAMUNIQ EN CULTURAL ARTE Y CULTURA.}

Exposiciones en ferias educativas del cantón Quevedo

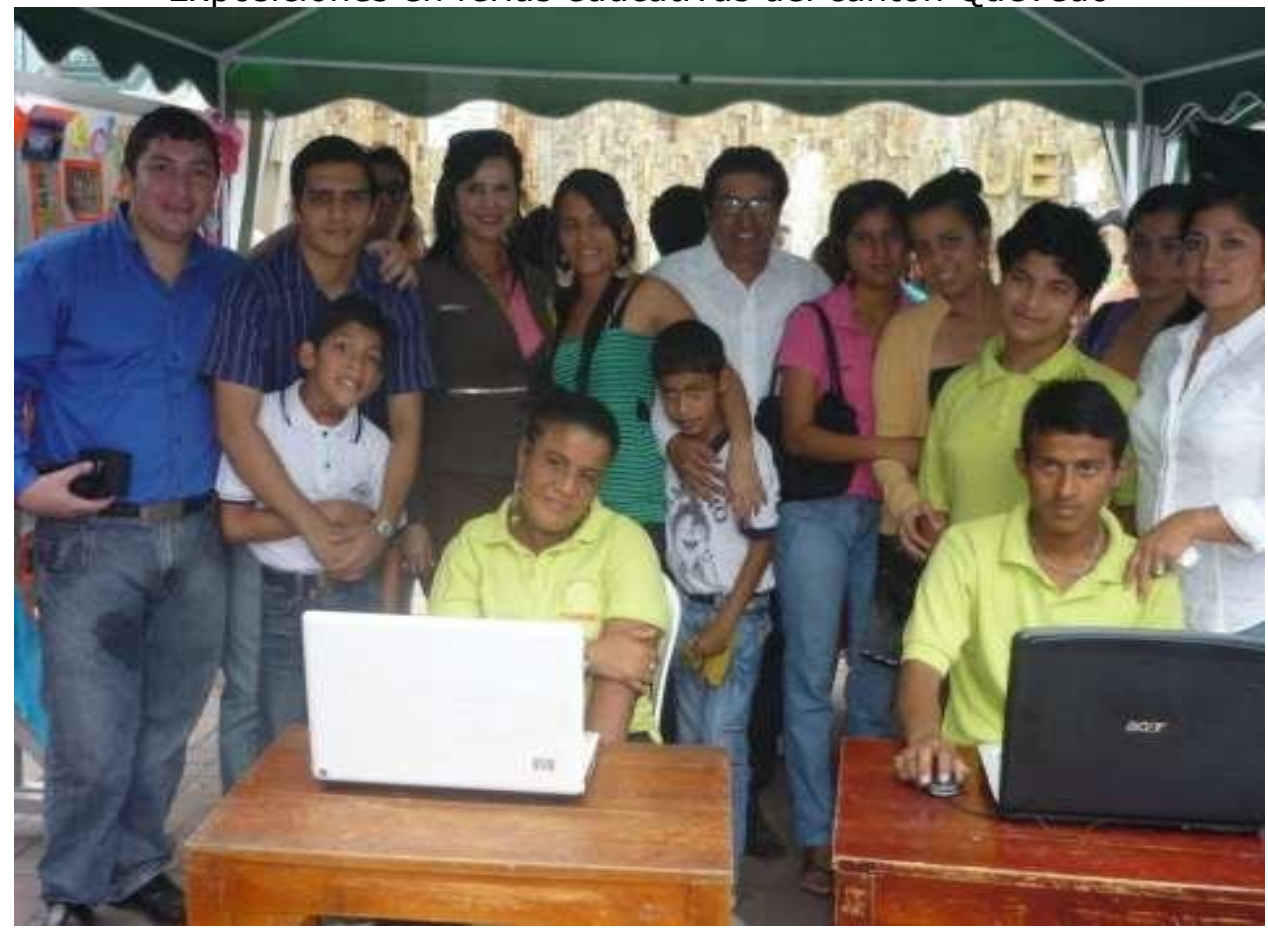

Niñas con Síndrome de Down participan en festivales

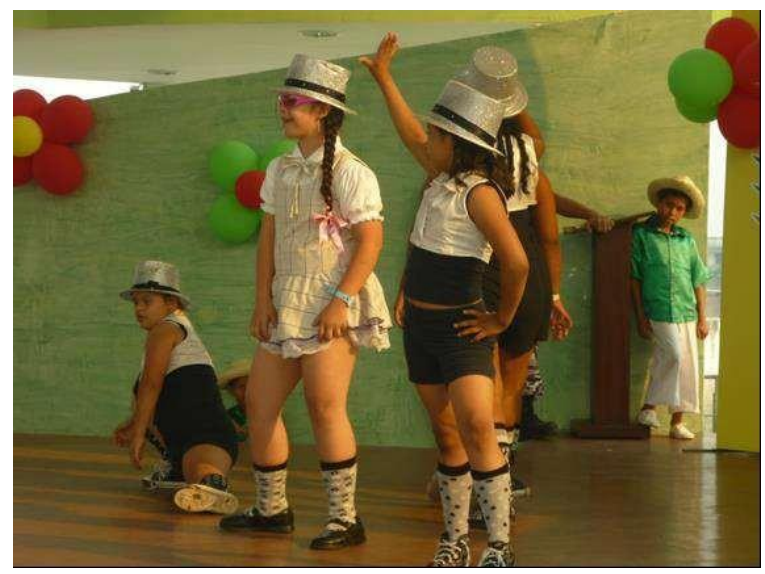

116

Revista Científica Ciencia y Tecnología. Vol 18 No 20, 2018 / págs. 101 -121 
No. 20, 31 de octubre de 2018

ISSN impreso: 1390 - 6321

En diciembre del 2011 se realizó el primer concurso interno de dibujo y pintura

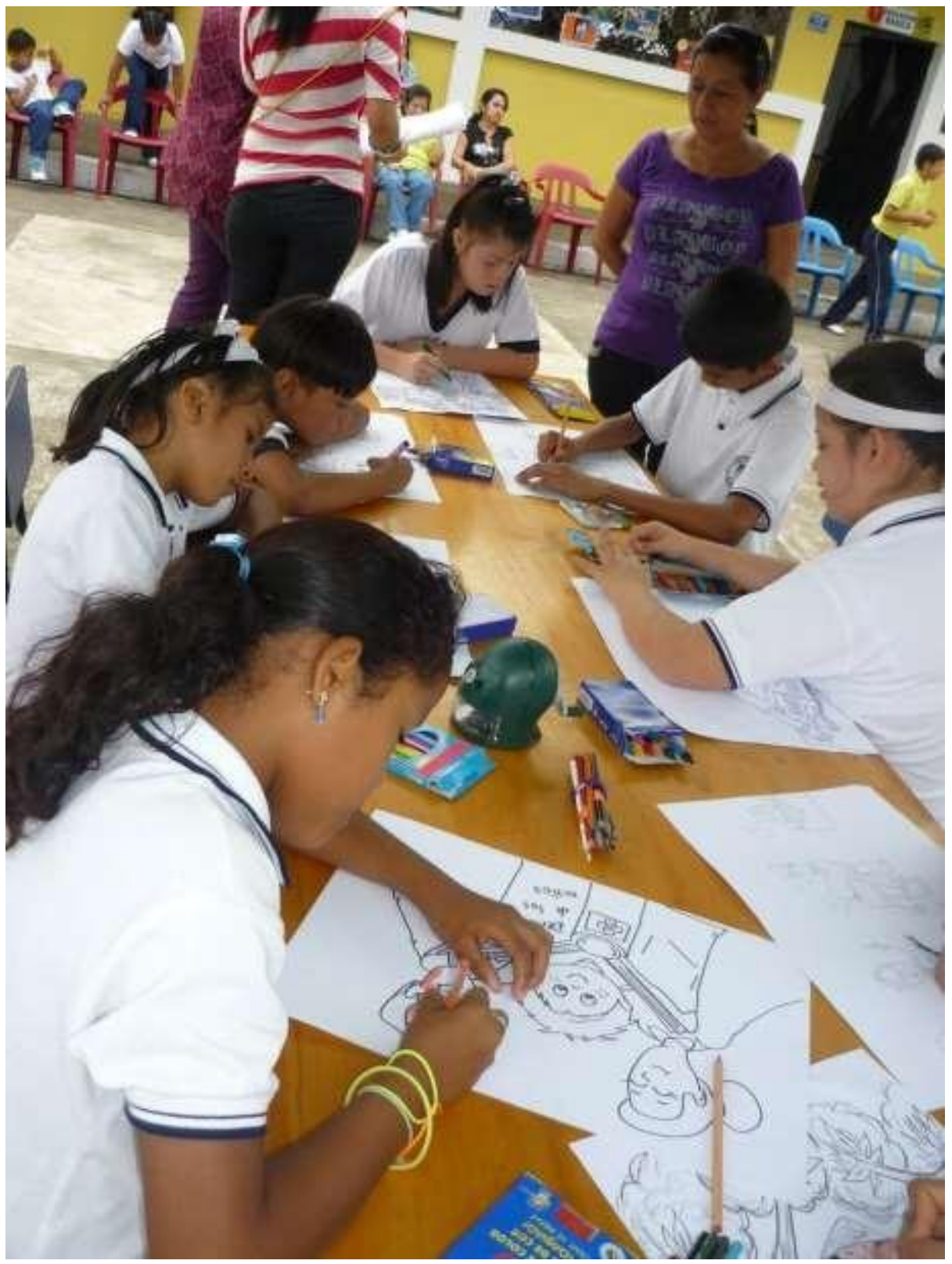

Bajaña Torres, Veliz, Osorio, Gómez, Martínez. Un cultivo resiliente para enfrentar el cambio climático la balsa (ochroma pyramidale sw) 


\section{Fomentando el desarrollo deportivo PAMUNIQ.}

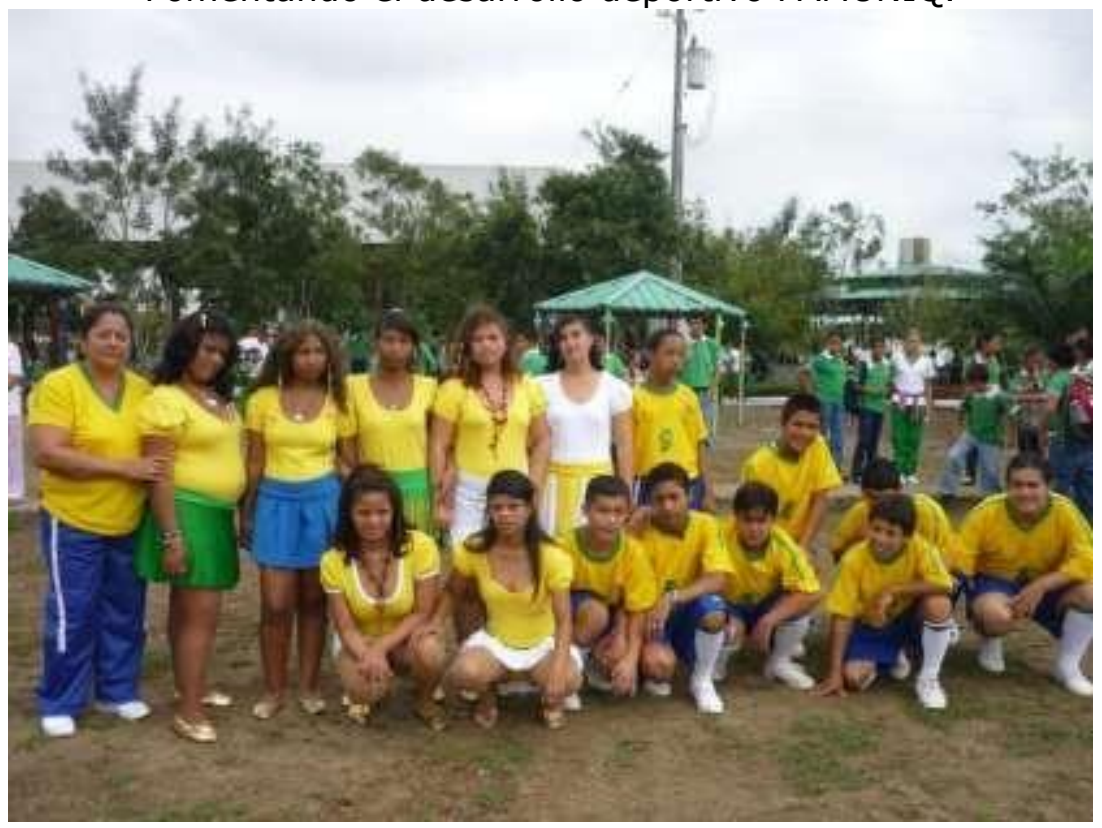

Campaña de Sensibilización 3 de diciembre del 2012

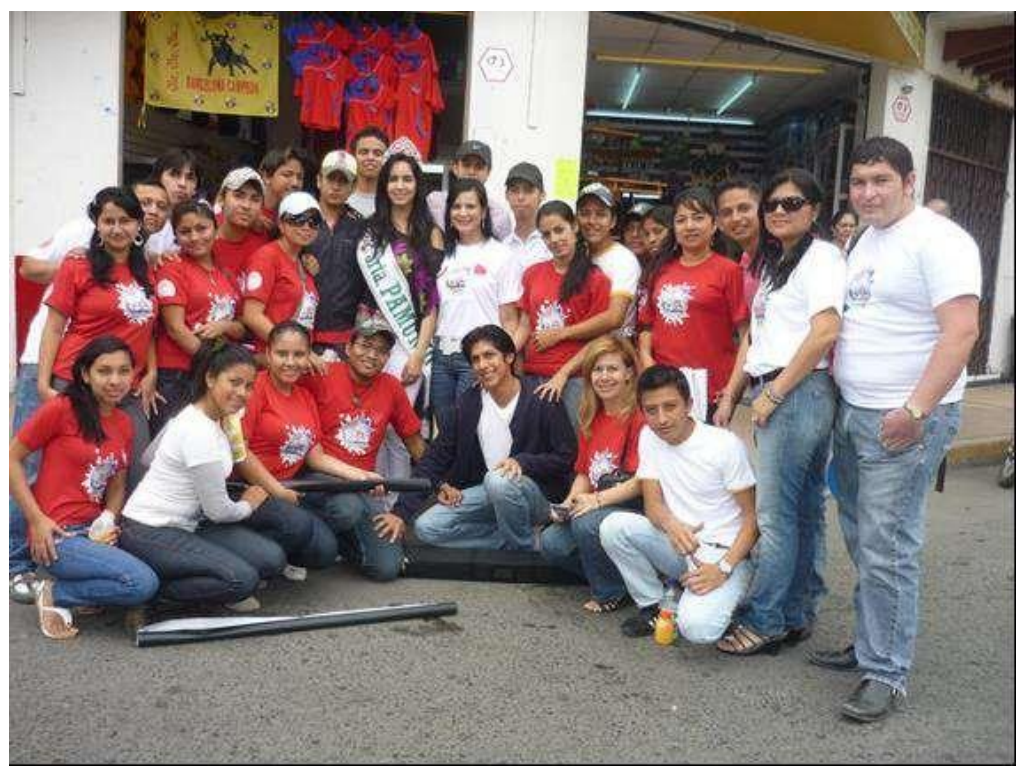

118

Revista Científica Ciencia y Tecnología. Vol 18 No 20, 2018 / págs. 101 -121 
No. 20, 31 de octubre de 2018

ISSN impreso: 1390 - 6321

Posicionamiento de la Imagen Corporativa de Pamuniq difundiendo los servicios que ofrece.

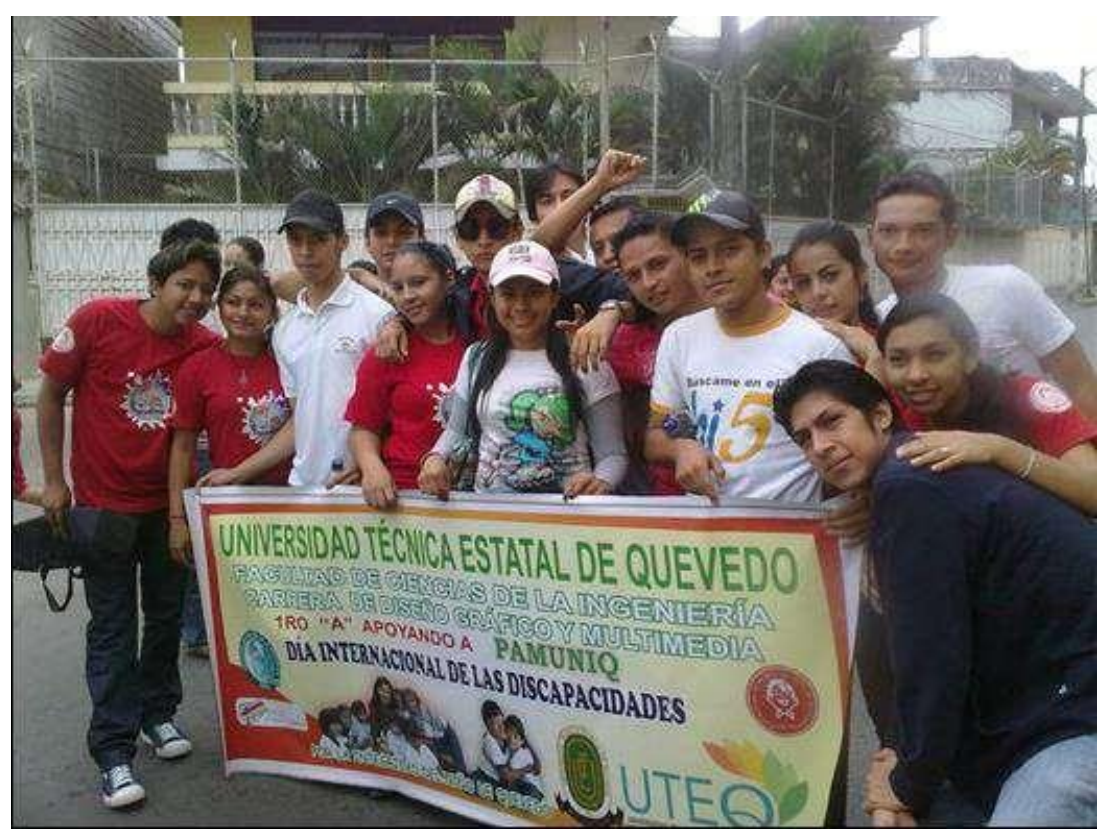

Los alumnos Diseño Gráfico UTEQ diseñaron la imagen corporativa del Patronato Municipal del niño de Quevedo

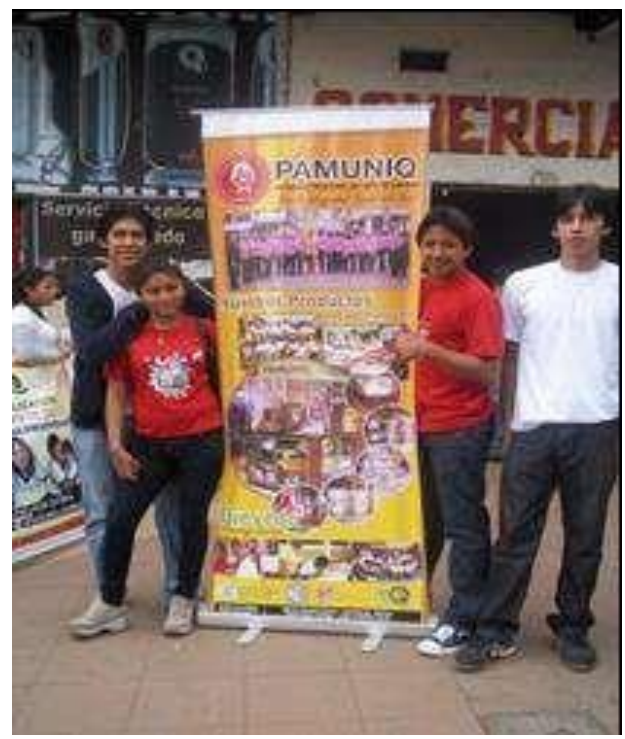

119

Bajaña Torres, Veliz, Osorio, Gómez, Martínez. Un cultivo resiliente para enfrentar el cambio climático la balsa (ochroma pyramidale sw) 
Recursos didácticos elaborados por el estudiante Javier Gómez como producto del trabajo de investigación.

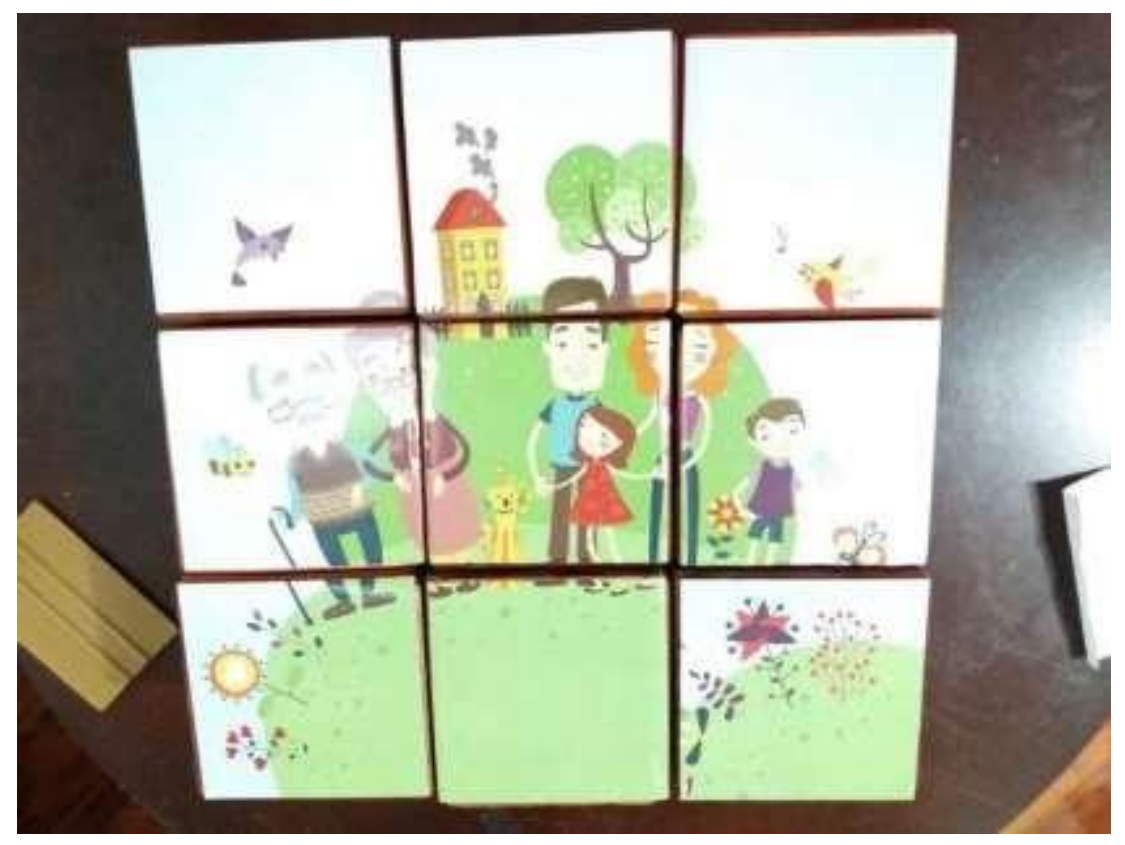

Niña con discapacidad intelectual juegan con los recursos didácticos

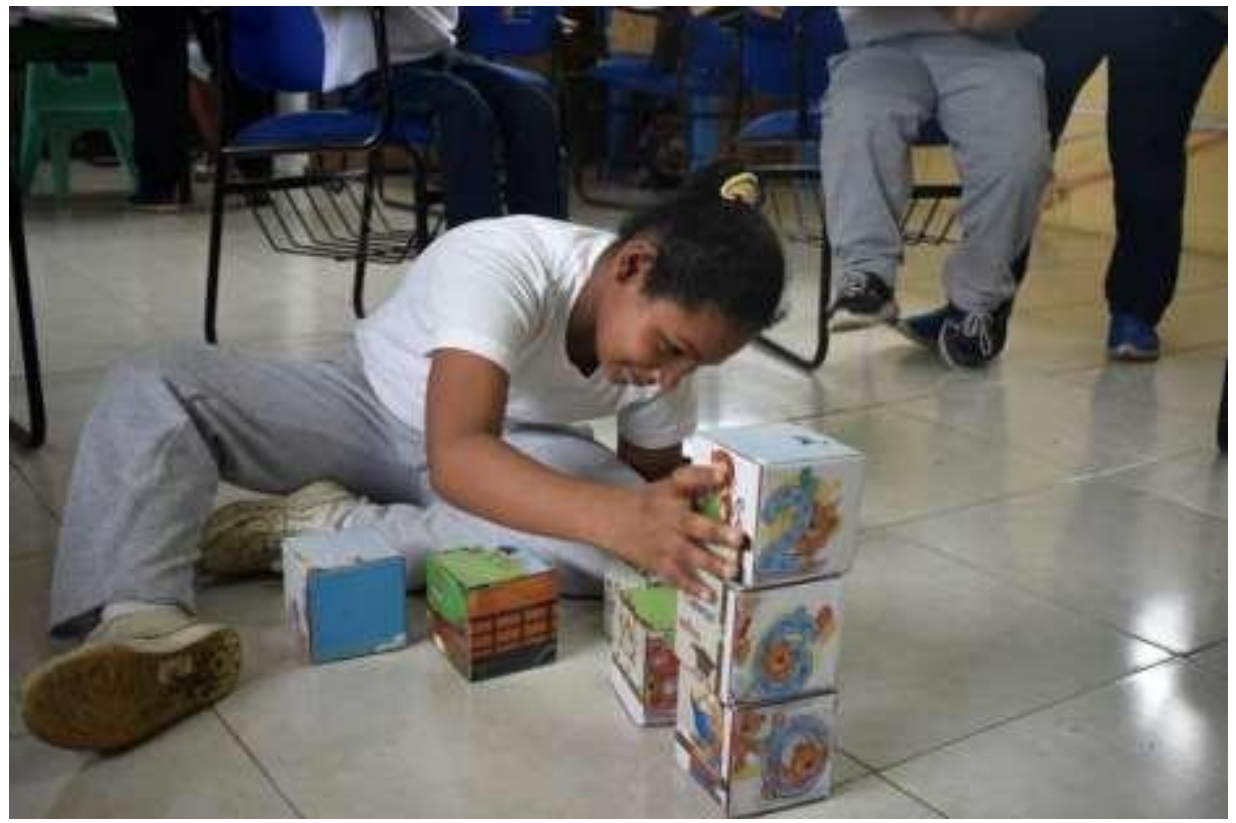

120

Revista Científica Ciencia y Tecnología. Vol 18 No 20, 2018 / págs. 101 -121 
Festival de danza ecuatoriana participando los niños de Pamuniq

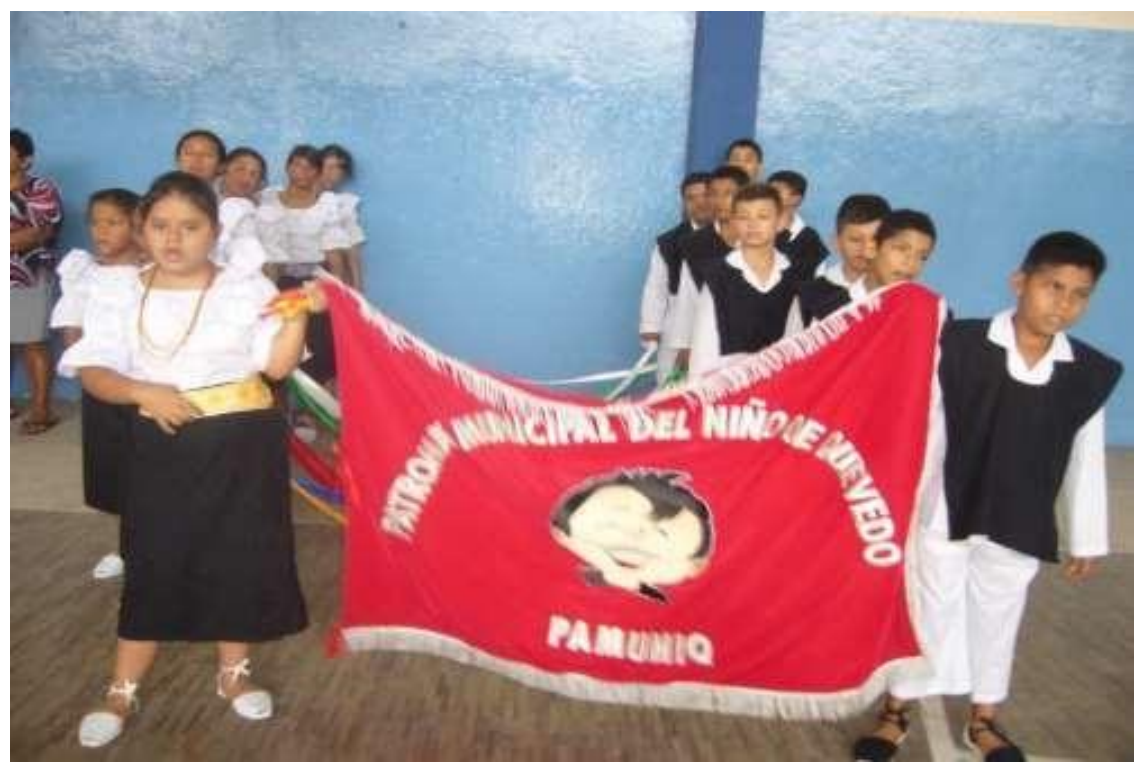
enfrentar el cambio climático la balsa (ochroma pyramidale sw) 